\title{
Palm Print Edge Extraction Using Fractional Differential Algorithm
}

\author{
Chunmei Chi and Feng Gao \\ Computer Engineering School, Qingdao Technological University, Qingdao 266033, China \\ Correspondence should be addressed to Feng Gao; gaofeng99@sina.com
}

Received 22 January 2014; Accepted 10 March 2014; Published 10 April 2014

Academic Editor: Gongzhu Hu

Copyright ( 2014 C. Chi and F. Gao. This is an open access article distributed under the Creative Commons Attribution License, which permits unrestricted use, distribution, and reproduction in any medium, provided the original work is properly cited.

\begin{abstract}
Algorithm based on fractional difference was used for the edge extraction of thenar palm print image. Based on fractional order difference function which was deduced from classical fractional differential G-L definition, three filter templates were constructed to extract thenar palm print edge. The experiment results showed that this algorithm can reduce noise and detect rich edge details and has higher SNR than traditional methods.
\end{abstract}

\section{Introduction}

Thenar area refers to the uplift muscle which is the joint of one's wrist and thumb. Everyone has his or her unique thenar palm print patterns. But the palm print texture structure changes with age and disease. For example, roughness and thickness appear in the palm print with disease. According to the Traditional Chinese Medicine theory, one's thenar palm print image is closely related to certain diseases such as asthma. For some patients, lattice distribution and rough texture appear in their thenar palm print. Doctors tend to think that the shape of one's palm print is closely related to his or her kidney deficiency and could be an indicator of asthma and can be used for the diagnosis of some allergic disease. In the medical practice of Traditional Chinese Medicine, by observing the patient's thenar palm print direction, the distance between palm print lines, groove depth, grain interaction, and pattern characteristic, doctors usually divide the thenar palm print into 4 levels. Class A: thenar skin is moist and soft with delicate texture and furrow is very shallow; no distribution characteristic pattern can be seen. Class B: thenar skin is moist and soft, texture is clear, and lattice type distribution can be found, but the gap is narrow. Class C: thenar skin is smooth but less moist, texture is clear and visible, lattice type distribution can be found, and the gap is wider. Class D: thenar skin is dry and rough, texture is clear and visible, large lattice type distribution can be found, and texture space is significantly widened compared with the Class C level. So far, the classification of thenar palm print of patients relies on the subjective observation of the doctors' naked eyes. In addition to medical treatment, palm print is also studied for other purposes [1-4].

In order to make the observation more objective and more accurate, we should conduct preprocessing, feature extraction and classification of the thenar palm print by using image processing technology. The computing is particularly important in order to realize the quantitative identification of thenar palm print. Thenar palm print image edge mainly refers to the texture features, such as lines, the distance between the lines, and the pattern characteristic, which is the main basis to carry out further classification. So effective edge detection plays an important role in feature extraction and quantitative identification. Edge detection (see [5-14]) method can detect the image edge to the precise location of thenar palm print and can also inhibit irrelevant details and noise. The classic methods of image edge detection like gradient method, conventional first-order differential and two-order differential method, and Laplace differential 
algorithm are sensitive to noise. Their performance for the thenar palm print image edge extraction is not accurate and could not obtain the edge localization and achieve noise filtering. Generally speaking, the low frequency part of the image corresponds to the smooth area, namely, the image of the nonedge region, while the middle frequency part of the image corresponds to texture information and the high frequency part corresponds to the image edge area and noise.

With regard to the thenar palm print image processing, if an integer order differential method is employed, texture details with little gray changes in the palm print image will decay greatly. If fractional differential algorithm is employed, this information can be retained to some extent. So compared with integer order differential method, the fractional differential method is in a better position to deal with the high frequency texture image. As for the thenar palm print image, texture information is what the doctors are really interested in. So the edge detection algorithm using fractional order differential characteristics of palm print is preferred. In this paper, first, the fractional difference equation is obtained according to G-L fractional differential definition. Second, we construct some filter templates to extract the palm print image edge. The experiments show that the fractional differential edge detection algorithm is better than integer order differential methods and also has higher signal-to-noise ratio.

\section{Fractional Differential Operators}

2.1. Fractional Calculus. Fractional calculus should really be called noninteger order calculus. As early as nineteenth century, the concept of fractional calculus appeared together with integer order calculus. In the past two hundred years, fractional calculus has gradually developed into a system as a pure mathematical branch. But not until recent decades, and with the development of engineering technology, has fractional calculus caused the attention of both scientists and engineers. It was found that the fractional differential operator has many advantages over integer order ones in many engineering problems. Many scholars have developed different definitions of fractional calculus, among which the most well-known definitions are Riemann-Liouville's definition and Caputo's definition $[15,16]$. These definitions are equivalent to each other in most cases. But GrunwaldLetnikov's definition is easier to be applied in numerical analysis. In this paper, we use Grunwald-Letnikov's definition to construct fractional order differential filter for image filtering and edge extraction of thenar palm print.

2.2. Fractional Difference. Fractional differential definition is a kind of generalization of the classical integer order derivative definition for continuous function. Usually, the $n$th derivative of a continuous function is defined as

$$
\frac{d^{n} f(x)}{d x^{n}}=\lim _{h \rightarrow 0} \frac{1}{h^{n}} \sum_{i=0}^{n}(-1)^{i}\left(\begin{array}{c}
n \\
i
\end{array}\right) f(x-i h) .
$$

The above differential operator does not need to be constrained only to integers. For example, the $(1+i)$ th derivative of the $(1-i)$ th derivative yields the 2 nd derivative.

For a general function $f(x)$ and $0<\alpha<1$, the complete fractional derivative is

$$
D^{n} f(x)=\frac{1}{\Gamma(1-\alpha)} \frac{d}{d x} \int_{0}^{x} \frac{f(t)}{(x-t)^{\alpha}} d t .
$$

For arbitrary $\alpha$, because the gamma function is undefined for arguments whose real part is a negative integer, it is necessary to apply the fractional derivative after the integer derivative has been performed. For example,

$$
D^{3 / 2} f(x)=D^{1 / 2} D^{1} f(x)=D^{1 / 2} \frac{d}{d x} f(x) .
$$

Generally, we have Riemann-Liouville fractional derivative

$$
D_{t}^{\alpha} f(t)=\frac{d^{n}}{d t^{n}} D_{t}^{-(n-\alpha)} f(t)=\frac{d^{n}}{d t^{n}} I_{t}^{n-\alpha} f(t) .
$$

The corresponding derivative is calculated using Lagrange's rule for differential operators. Computing $n$th order derivative over the integral of order $n-\alpha$, the $\alpha$ order derivative is obtained. It is important to remark that $n$ is the nearest integer bigger than $\alpha$.

Caputo Fractional Derivative. There is another way for defining fractional derivatives, the Caputo fractional derivative which was introduced by $M$. Caputo. In contrast to the Riemann Liouville fractional derivative, when solving differential equations using Caputo's definition, it is not necessary to define the fractional order initial conditions. Caputo's definition is as follows:

$$
{ }_{a}^{C} D_{t}^{\alpha} f(x)=\frac{1}{\Gamma(n-\alpha)} \int_{a}^{x} \frac{f^{(n)}(t)}{(x-t)^{\alpha+1-n}} d t .
$$

Besides the above two definitions, we have also Grunwald-Letnikov's fractional derivative definition as shown below by using $\Gamma$ function:

$$
\frac{d^{\alpha} f(x)}{d x^{\alpha}}=\lim _{h \rightarrow 0} \frac{1}{h^{\alpha}} \sum_{i=0}^{\infty}(-1)^{i} \frac{\Gamma(\alpha+1)}{\Gamma(\alpha-i+1)} f(x-i h),
$$

where

$$
\Gamma(n)=\int_{0}^{\infty} t^{n-1} \cdot e^{-t} d t=(n-1) !
$$

and $\alpha$ can be noninteger. The $\alpha$ th difference of $f(x)$ can be expressed as follows:

$$
\begin{aligned}
f^{\alpha}(x) \approx & f(x)+(-\alpha) f(x-1)+\frac{(-\alpha)(-\alpha+1)}{2} \\
& \cdot f(x-2)+\frac{(-\alpha)(-\alpha+1)(-\alpha+2)}{3 !} f(x-3) \\
& +\cdots+\frac{\Gamma(-\alpha+1)}{n ! \Gamma(-\alpha+n+1)} f(x-n)
\end{aligned}
$$

The $\alpha$ th difference is actually the approximation of the $\alpha$ th derivative. Fractional calculus has been applied to many areas [15-20]. 


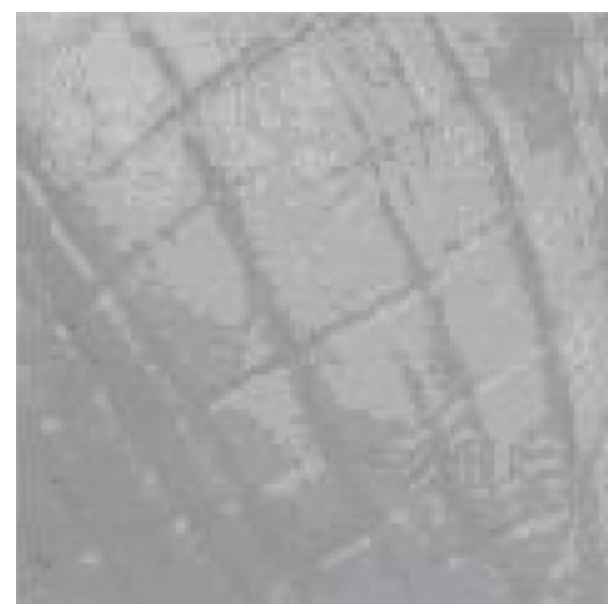

FIgURE 1: The original image.

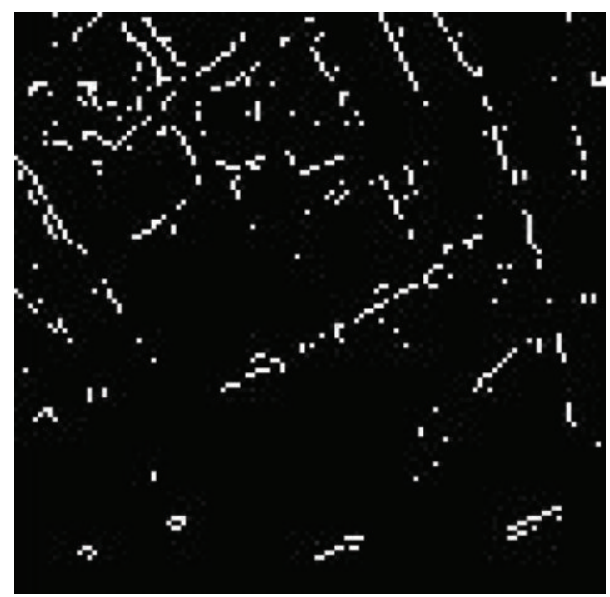

FIGURE 2: Image got by Sobel operator.

2.3. Fractional Order Filter. In general, the $M \times N$ image filter template for a gray image is given as follows:

$$
g(x, y)=\sum_{s=-a}^{a} \sum_{t=-b}^{b} w(s, t) f(x+s, y+t)
$$

where $a=(m-1) / 2$ and $b=(n-1) / 2$.

In order to get a complete picture of the filtered image, the filter template must be applied to $X=0,1, \ldots, M-1$ and $Y=0,1, \ldots, N-1$. Thus, we guarantee that all the pixels in the image are filtered. For digital images, the fractional order differential filter can be equal to the image itself in size. In order to realize the filtering and minimize the error, we can select the first three terms in the fractional difference to construct isotropic filter. We construct the below fractional order differential filter template 1 , filter template 2 , and filter template 3. In the structure of filter 3, we first consider the $X$ direction and $Y$ direction around the upper and lower side. So there are two $f(x, y)$ terms in the two directions; therefore, there are four $f(x, y)$ terms. Considering the effect of oblique direction of 45 degrees above the pixels, we add 4 oblique

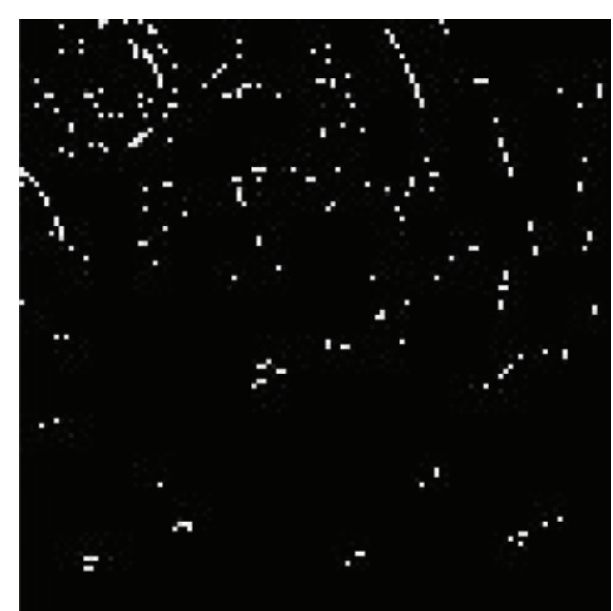

Figure 3: Image got by Prewitt operator.

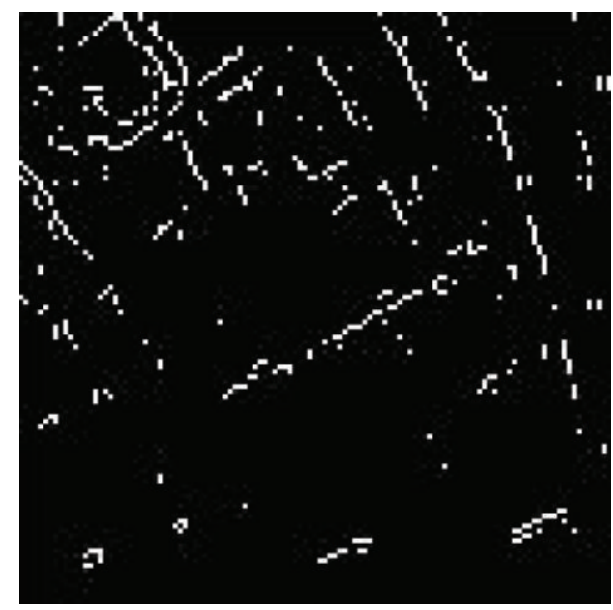

FIGURE 4: Image got by Laplace operator.

TABLE 1: Filter template 1.

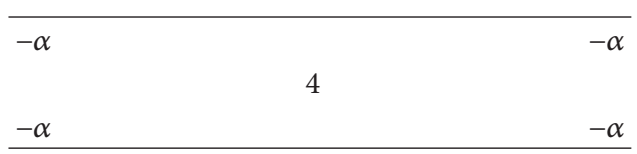

TABLE 2: Filter template 2.

\begin{tabular}{lcc}
\hline$-\alpha$ & $-\alpha$ & $-\alpha$ \\
$-\alpha$ & 8 & $-\alpha$ \\
$-\alpha$ & $-\alpha$ & $-\alpha$ \\
\hline
\end{tabular}

directions. Because each oblique above contains one $f(x, y)$ term, there are $8 f(x, y)$ terms for all the different directions.

All the fractional order differential filter templates have the same direction of rotation (see Tables 1, 2, and 3).

\section{Image Edge Extraction Experiments}

Experiments were carried out by using MATLAB. The thenar palm print image was taken by the digital camera in high 


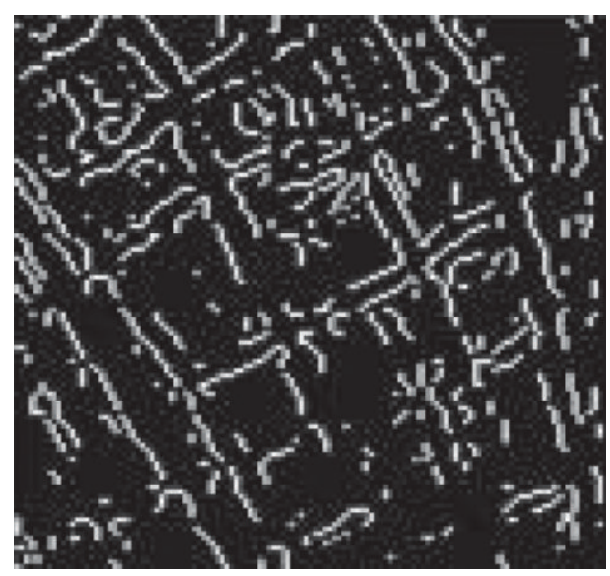

FIGURE 5: Edge extracted by Sobel operator.

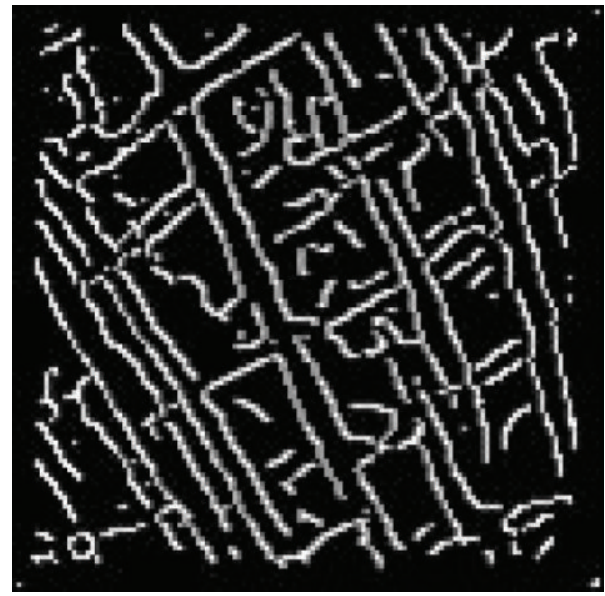

Figure 6: Edge extracted by Prewitt operator.

TABle 3: Filter template 3.

\begin{tabular}{ccccc}
\hline$\frac{\alpha * \alpha-\alpha}{2}$ & & $\frac{\alpha * \alpha-\alpha}{2}$ & & $\frac{\alpha * \alpha-\alpha}{2}$ \\
$\frac{\alpha * \alpha-\alpha}{2}$ & $-\alpha$ & $-\alpha$ & $-\alpha$ & \\
$\frac{\alpha * \alpha-\alpha}{2}$ & $-\alpha$ & 8 & $-\alpha$ & $\frac{\alpha * \alpha-\alpha}{2}$ \\
\hline
\end{tabular}

light background. The digital camera's shooting angle is perpendicular to the palm thenar area and the palm is in the natural state of relaxation. Firstly, we carry out the palm print image segmentation to identify the thenar palm print region by using palm print positioning point segmentation method based on the segmentation of the thenar palm print image. Second, we use the three-edge detection method to process the thenar palm print image. The test results are shown in Figures 1, 2, 3, 4, 5, 6, 7, 8, 9, 10, 11, 12, 13, 14, and 15. For comparison, we also use the traditional integer order differential operator Sobel, Roberts, Prewitt, LOG, and Laplace operator to construct filter templates to process the

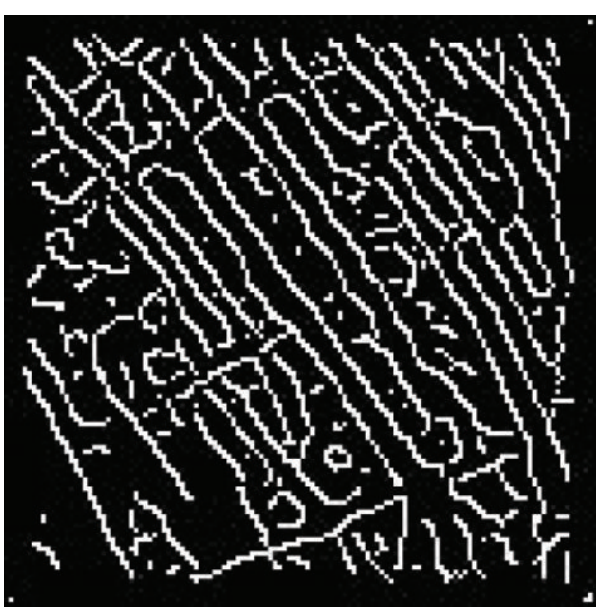

FIGURE 7: Edge extracted by Laplace operator.

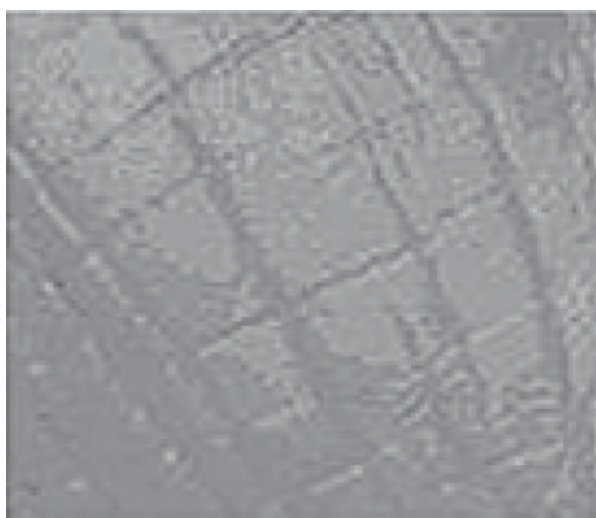

FIGURE 8: Image got by 0.9 th template 1 .

thenar palm print image. The traditional edge detection is mainly to estimate the direction gradient of gray image by scale change detector of gradient operator or derivative based on these changes. We enhance the area in the image and then carry out the threshold operation on the gradient. If the gradient value is greater than a given threshold, then there is edge. Test effect is shown below. It can be seen from Figure 1 that different filter templates yield different palm print edge intensities.

3.1. Analysis of Fractional Order on the Processing Results. The experiment analyses of filter template 1 , filter template 2 , and filter template 3 show that when the fractional order is 0.5 , the palm print image is clear and with least noise. With the elevation of fractional order, palm print texture details become clear and rich gradually. As the filter size increases, the edge becomes clear, but not very obvious.

3.2. Comparison of Fractional Differential Algorithm with Other Edge Extract Operators. The image in Figure 5 is the classical first-order Sobel operator, Prewitt operator, and the second-order Laplacian operator and fractional order $(0.5$ order) comparison diagram edge extraction operator. The 


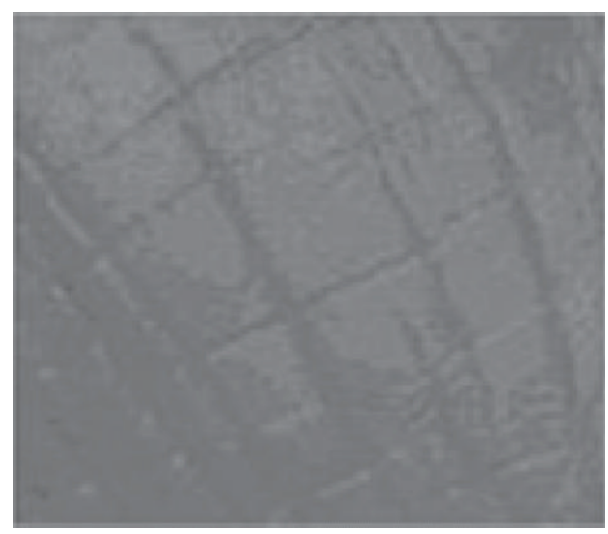

FIGURE 9: Image got by 0.8 th template 1 .

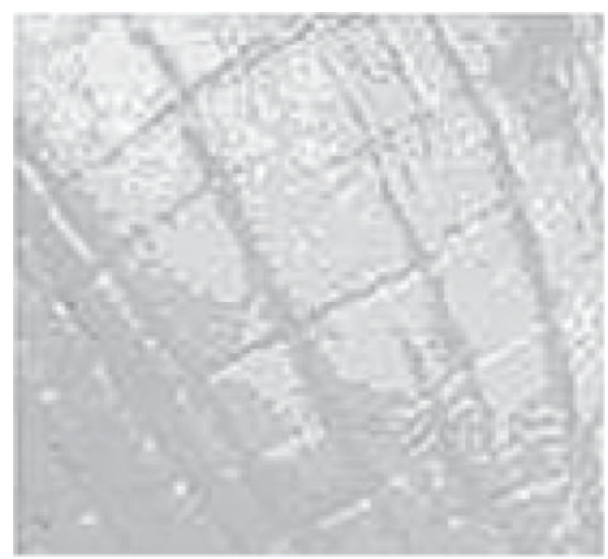

FIGURE 10: Image got by 0.7 th template 1 .

diagram shows that fractional templates detect edge detail and localization accurately.

3.3. The Peak Signal-to-Noise Ratio for Different Algorithms. We add random noise to palm print image and compare the peak signal-to-noise ratio (PSNR) for various algorithms to extract edge information. The peak signal-to-noise ratio (PSNR) formula is

$$
\operatorname{psnr}\left(f_{0}, f\right)=10 \log _{10} \frac{N \times M \times 255^{2}}{\sum_{j=1}^{M} \sum_{i=1}^{N}\left[f(i, j)-f_{0}(i, j)\right]^{2}} .
$$

The higher the peak signal-to-noise ratio (PSN) is, the better the effect to extract texture information is. We adopt the following evaluation method. We first do image edge extraction with adding random noise and without noise by using different methods. Then we analyze the difference between the two methods. In the above equation, $f(x, y)$ represents image edge extracted from the noise added image.

In Table 1, the peak signal-to-noise ratio of different order differential extractions based on edge information is listed where the stochastic noise with mean value 0 and variance 0.003 was added. And from the result we can see that the

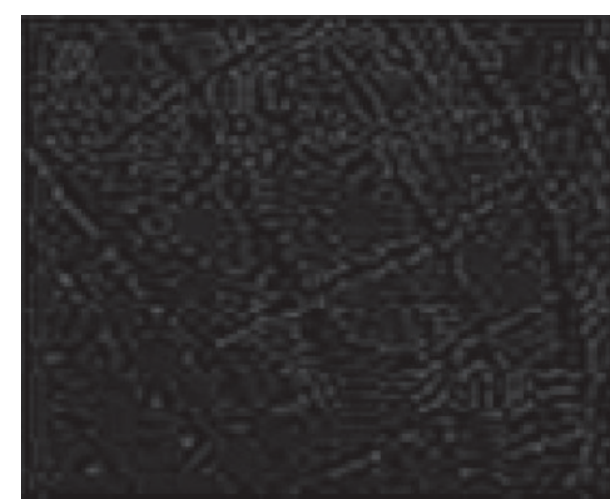

FIgURE 11: Image got by 0.6 th template 1 .

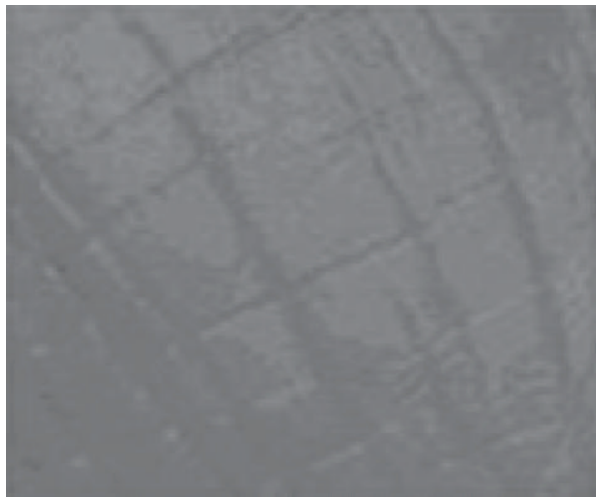

FIGURE 12: Image got by 0.1 th template 2 .

TABLE 4: PSNR of edge information extracted by different order differential.

\begin{tabular}{lcccccc}
\hline \multirow{2}{*}{ Number of iter. } & \multirow{5}{c}{ Sob } & Lap & \multicolumn{5}{c}{ Fethod } \\
& & & 0.6 & 0.4 & 0.2 & 0.1 \\
\hline 1 & 22.55 & 22.40 & 23.15 & 23.36 & 23.55 & 22.74 \\
2 & 23.7 & 23.81 & 24.47 & 24.56 & 24.63 & 24.15 \\
\hline
\end{tabular}

antinoise performance of differential algorithm is good and can be used to extract the edge of thenar palm print image.

In Table 4, we list the peak signal-to-noise ratio for different methods to extract the edge of image.

From Table 4, it can be seen that although the impact of the two added noises is different, the signal-to-noise ratio of the differential algorithm is higher than that of Sobel method and Laplace method. And the peak signal-to-noise ratio decreases with the increase of differential order. The order of peak signal-to-noise ratio and the maximum value appeared in the 0.2 order. When the order is less than 0.2 , the peak signal-to-noise ratio began to decrease. If the differential order is very small, the fractional algorithm has no significant effect on the enhancement of palm print texture; meanwhile, the image texture information has an effect on extraction of the edge. We also notice that the noise has different effects on different parts of the image. As shown in Table 1, for the mean value 0 and variance 0.003 random noise, the effect on the 


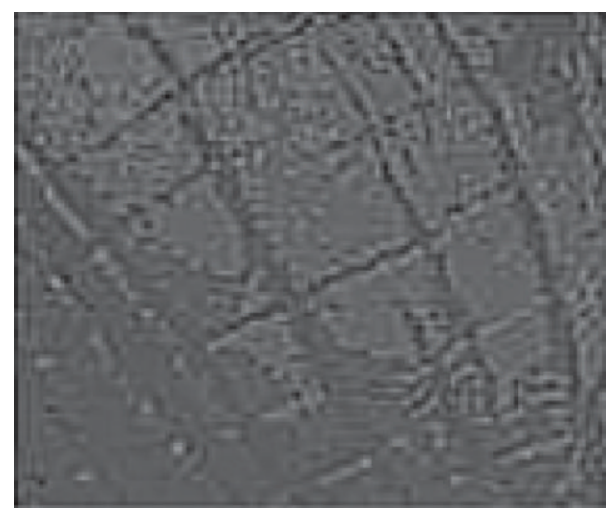

FIgURE 13: Image got by 0.9 th template 3 .

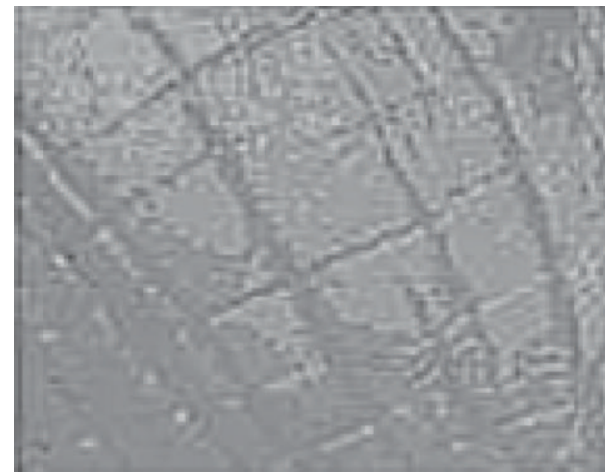

FIgURE 14: Image got by 0.8 th template 3 .

extraction of edge information is different. At the same time filter templates 1-3 are only an approximation of fractional order differential template, so we can only conclude that the optimal fractional order of differential template number is 0.2 .

\section{Conclusion}

Simulation results show that the ability of fractional differential edge detection algorithm to extract the image edge information varies from different fractional orders. In general, the ability gradually increases with the increasing of order. The ability also increases with the template size but not in a positive proportional manner. Compared with the traditional first-order and second-order differential operators, the edge detection based on fractional differential method has higher signal-to-noise ratio. In this paper, the template itself is actually the approximate representation of a fractional differential method. So how to find the fractional order differential operators to achieve higher accurate edge extraction is a problem for further study.

\section{Conflict of Interests}

The authors declare that there is no conflict of interests regarding the publication of this paper.

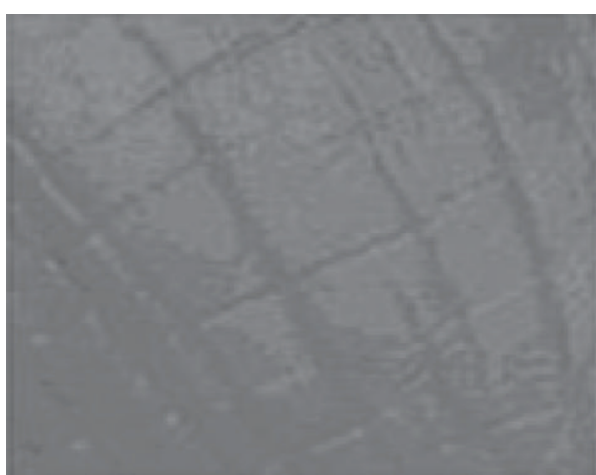

Figure 15: Image got by 0.7 th template 3 .

\section{Acknowledgment}

This work is supported by the National Natural Science Foundation of China, no. 31271077.

\section{References}

[1] D. Zhang, Palm Print Authentication, Kluwer Academic, New York, NY, USA, 2004.

[2] E. Liu, A. K. Jain, and J. Tian, "A coarse to fine minutiaebased latent palm print matching," IEEE Transactions on Pattern Analysis and Machine Intelligence, vol. 35, no. 10, pp. 2307-2322, 2013.

[3] J. Yao and W.-K. Cham, "3D modeling and rendering from multiple wide-baseline images by match propagation," Signal Processing: Image Communication, vol. 21, no. 6, pp. 506-518, 2006.

[4] E. Liu, H. Zhao, J. Liang, L. Pang, H. Chen, and J. Tian, "Random local region descriptor (RLRD): a new method for fixed-length feature representation of fingerprint image and its application to template protection," Future Generation Computer Systems, vol. 28, no. 1, pp. 236-243, 2012.

[5] H. Jeong and C. I. Kim, "Adaptive determination of filter scales for edge detection," IEEE Transactions on Pattern Analysis and Machine Intelligence, vol. 14, no. 5, pp. 579-585, 1992.

[6] T. Aydin, Y. Yemez, E. Anarim, and B. Sankur, "Multidirectional and multiscale edge detection via M-band wavelet transform," IEEE Transactions on Image Processing, vol. 5, no. 9, pp. 13701377, 1996.

[7] A. P. Paplinski, "Directional filtering in edge detection," IEEE Transactions on Image Processing, vol. 7, no. 4, pp. 611-615, 1998.

[8] C.-J. Sze, H.-Y. M. Liao, H.-L. Hung, K.-C. Fan, and J.-W. Hsieh, "Multiscale edge detection on range images via normal changes," IEEE Transactions on Circuits and Systems II: Analog and Digital Signal Processing, vol. 45, no. 8, pp. 1087-1092, 1998.

[9] C. F. Olson, "Adaptive-scale filtering and feature detection using range data," IEEE Transactions on Pattern Analysis and Machine Intelligence, vol. 22, no. 9, pp. 983-991, 2000.

[10] D. Demigny, "On optimal linear filtering for edge detection," IEEE Transactions on Image Processing, vol. 11, no. 7, pp. 728737, 2002.

[11] S. Konishi, A. L. Yuille, J. M. Coughlan, and S. C. Zhu, "Statistical edge detection: learning and evaluating edge cues," IEEE Transactions on Pattern Analysis and Machine Intelligence, vol. 25, no. 1, pp. 57-74, 2003. 
[12] J. Wu, Z. Yin, and Y. Xiong, “The fast multilevel fuzzy edge detection of blurry images," IEEE Signal Processing Letters, vol. 14, no. 5, pp. 344-347, 2007.

[13] P. Bao, L. Zhang, and X. Wu, "Canny edge detection enhancement by scale multiplication," IEEE Transactions on Pattern Analysis and Machine Intelligence, vol. 27, no. 9, pp. 1485-1490, 2005.

[14] B. Mathieu, P. Melchior, A. Oustaloup, and C. Ceyral, "Fractional differentiation for edge detection," Signal Processing, vol. 83, no. 11, pp. 2421-2432, 2003.

[15] K. B. Oldham and J. Spanier, The Fractional Calculus, Academic Press, New York, NY, USA, 1974.

[16] A. A. Kilbas, H. M. Srivastava, and J. J. Trujillo, Theory and Applications of Fractional Differential Equations, Elsevier, Amsterdam, The Netherlands, 2006.

[17] S. W. Wheatcraft and M. M. Meerschaert, "Fractional conservation of mass," Advances in Water Resources, vol. 31, no. 10, pp. 1377-1381, 2008.

[18] W. Chen, H.-G. Sun, X. Zhang, and D. Korošak, "Anomalous diffusion modeling by fractal and fractional derivatives," Computers \& Mathematics with Applications, vol. 59, no. 5, pp. 1754$1758,2010$.

[19] H.-G. Sun, W. Chen, and Y.-Q. Chen, "Variable-order fractional differential operators in anomalous diffusion modeling," Physica A, vol. 388, no. 21, pp. 4586-4592, 2009.

[20] S. G. Samko, A. A. Kilbas, and O. I. Marichev, Fractional Integrals and Derivatives, Gordon and Breach, Yverdon, Switzerland, 1993. 


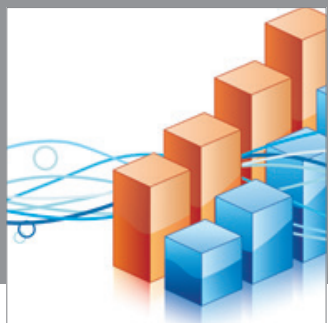

Advances in

Operations Research

mansans

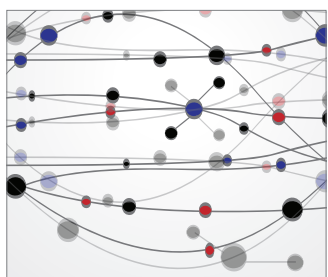

The Scientific World Journal
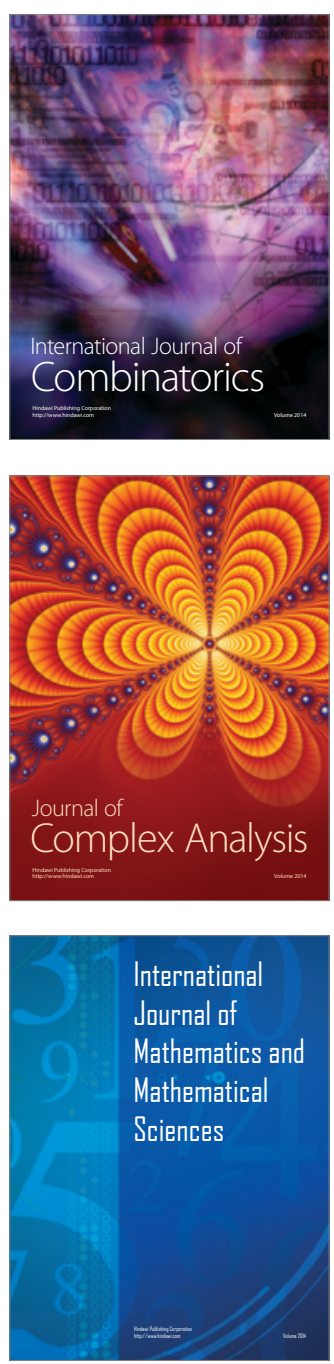
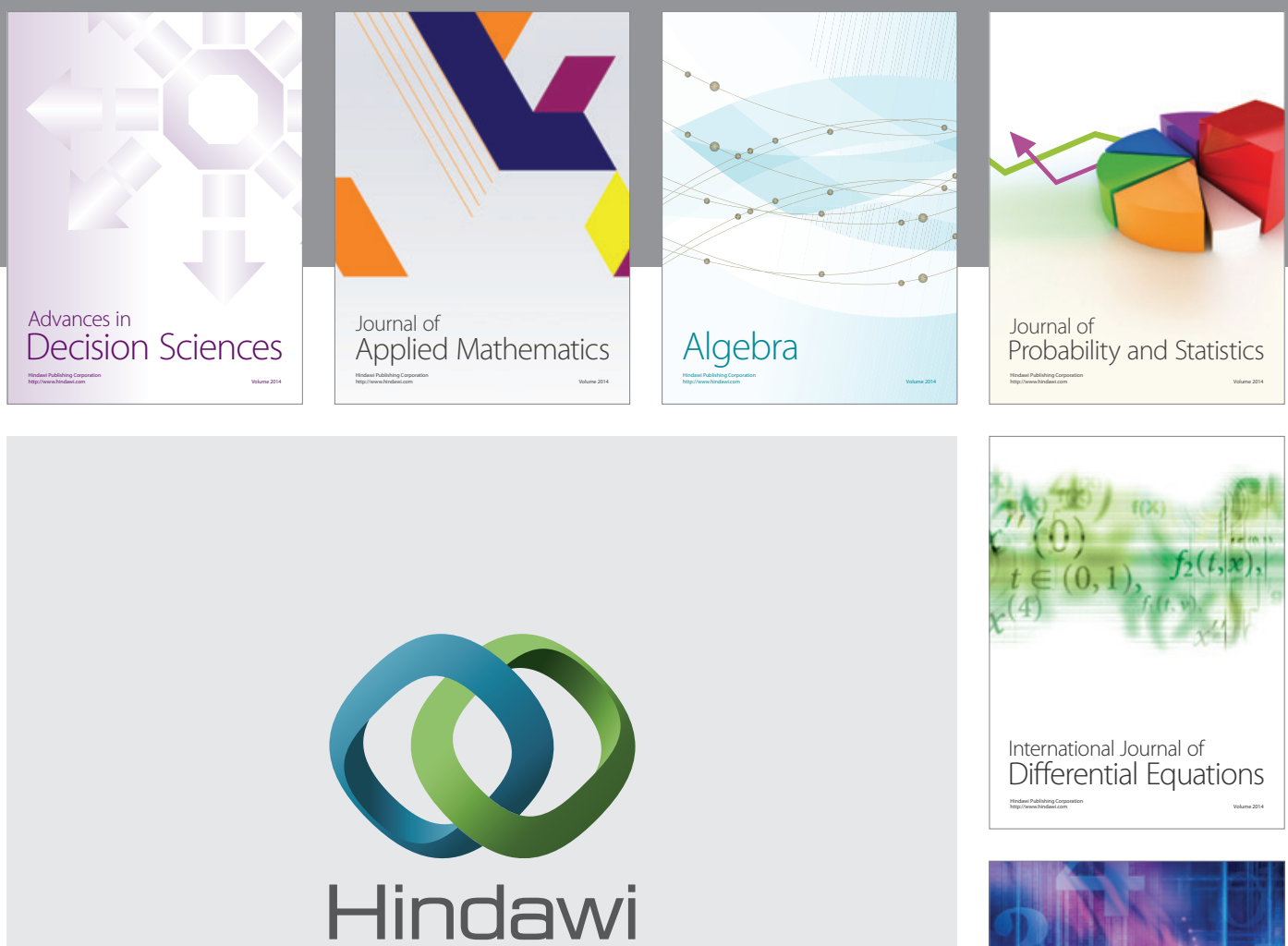

Submit your manuscripts at http://www.hindawi.com
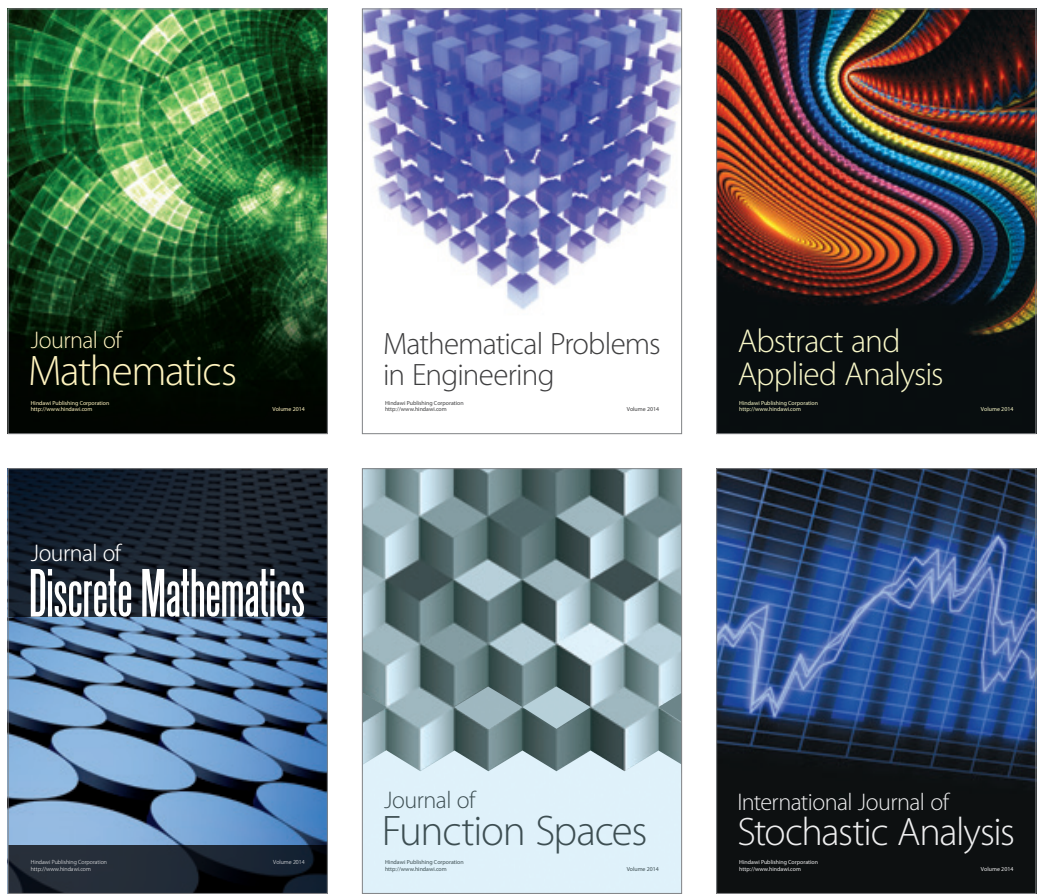

Journal of

Function Spaces

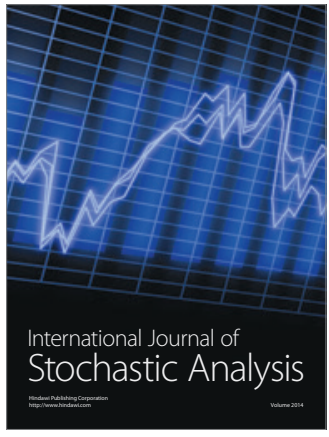

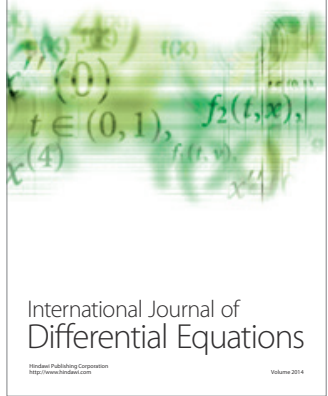
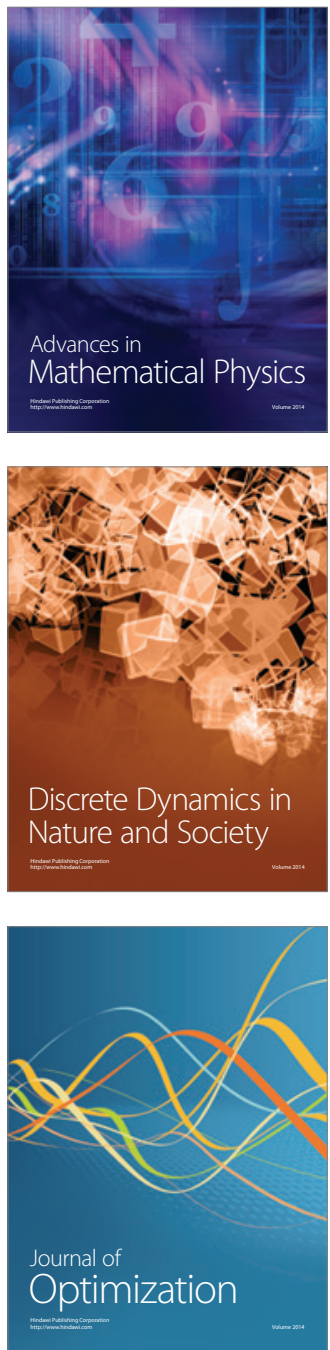